\title{
Prospecciones liquenológicas en África del Norte. III. Líquenes saxí- colas del cabo Tres Forcas (Nador, Marruecos) y cabo Falcón (Orán, Argelia).
}

\author{
J. M. EGEA
}

\begin{abstract}
EGEA, J. M. (1988). Lichenological prospections in North Africa. III. Saxicolous lichens from cape Tres Forcas (Nador, Marruecos) and cape Falcon (Oran, Argelia). Collect. Bot. (Barcelona) 17(2): 183-189.

The results of floristic and phytosociologic studies on lichen in five localities near Nador (Morocco) and Oran (Argelia) are presented. 67 taxa, some of them of a very high interest in this region, and 4 lichenical communities have been indentified.
\end{abstract}

Keywords: Lichenes, chorology, sociology, North Africa.

\section{Resumen}

EgeA, J. M. (1988). Prospecciones liquenológicas en África del Norte. III. Líquenes saxícolas del cabo Tres Forcas (Nador, Marruecos) y cabo Falcón (Orán, Argelia). Collect. Bot. (Barcelona) 17(2): 183-189.

Se presentan los resultados florísticos y fitosociológicos del estudio de 5 localidades situadas en las proximidades de Nador (Marruecos) y Orán (Argelia). Se citan 67 táxones, varios de ellos de gran interés en la región, y 4 comunidades liquénicas.

Palabras clave: Líquenes, corología, sociología, Norte de África.

\section{INTRODUCCIÓN}

Basamos este estudio en las recolecciones efectuadas en el Cabo Tres Forcas (Marruecos) en abril de 1984 y 1985 y en los alrededores del Cabo Falcón (Argelia) en abril de 1985.

La flora liquénica de este área era, hasta la fecha, prácticamente desconocida. Los factores de tipo climático y litológico, sin embargo, nos hacían prever una fuerte relación entre la flora y vegetación liquénica de esta zona y la del S.E. de España, paralela a la que hay entre la vegetación vascular.

Este hecho nos llevó a organizar una exploración botánica a Marruecos que se realizó en abril de 1984. Uno de los objetivos principales era el estudio de los líquenes que crecen sobre 
las lavas del Cabo Tres Forcas, en Nador. En la primavera de 1985 realizamos una campaña liquenológica en Argelia. En esta ocasión se visitó, entre otras localidades, la zona comprendida entre el Cabo Falcón y el Cabo Lindles, en Orán, y se completó el estudio del Cabo Tres Forcas.

Los datos obtenidos en el estudio de estas localidades constituyen el núcleo de este trabajo. Están situadas en el litoral y son las siguientes:

1) Nador. Cabo Tres Forcas, $100-200 \mathrm{~m}$.

2) Nador. Entre Beni Enzar y Faknana, 80-100 m.

3) Orán. Ain el Turck. Cabo Falcón, 30-50 m.

4) Orán. Ain el Turck. Los Andaluces, $50 \mathrm{~m}$.

5) Orán. Ain el Turck. Cabo Lindlés, 5-20 m.

En las 4 primeras localidades, la roca corresponde a afloramientos volcánicos del mioceno. En el Cabo Lindlés son arenas cementadas (calcarenitas).

El clima es de tipo mediterráneo semiárido con precipitaciones que oscilan entre $300 \mathrm{~mm}$ (Cabo Falcón, $322 \mathrm{~mm}$ ) y 400 (Melilla, $388 \mathrm{~mm}$ ) y temperaturas medias anuales situadas en torno a los $18^{\circ} \mathrm{C}\left(\right.$ Cabo Falcón, $18^{\prime} 2^{\circ} \mathrm{C}$; Melilla, $\left.18^{\prime} 3^{\circ} \mathrm{C}\right)$. Las heladas son ocasionales y prácticamente inexistentes.

Todas las localidades se encuentran dentro del piso termomediterráneo semiárido, con inviernos suaves, donde encuentran su óptimo un cierto número de especies liquénicas xerófilas y termófilas. La vegetación potencial corresponde a la asociación Periploco laevigataeTetraclinidetum articulatae Benabid 1984, de óptimo en el Rif oriental y en el Oranesado (BENABID 1984).

\section{VEGETACIÓN LIQUÉNICA}

Las comunidades liquénicas inventariadas apenas si presentan diferencias notables con las estudiadas en el SE de España (LLIMONA,1975, EgEA \& LLIMONA, 1982, 1987, LLIMONA \& EGEA, 1984) y Tenerife (EGEA \& col.,1987).

Es de destacar que no detectamos la presencia de diversas asociaciones, como: Acarosporetum charidemi Llimona 1987, Buellio-Caloplacetum littorae Llimona \& Egea, 1984 y de otras asociaciones nitrófilas como Solenosporo-Diploicietum subcanescentis Egea \& Llimona 1987, Acarosporetum heufleurianae Llimona \& Egea 1987. Sin embargo, es probable que un estudio más detallado de la zona permita reconocer alguna de estas comunidades.

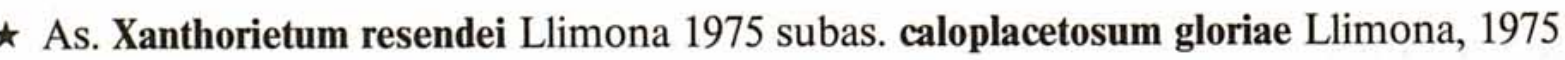

Comunidad de superficies soleadas localizada en Los Andaluces (W de Orán), sobre paredes subverticales que miran al mar. Se caracteriza por la gran abundancia local de Caloplaca gloriae (3.3), junto con otras especies características de la asociación como Xanthoria resendei (1.2), X. aureola (+), Buellia cerussata (1.1) Ramalina bourgeana (2.1) y Diploicia subcanescens (1.2). Es de destacar la ausencia de Dimelaena radiata, una de las especies más constantes en esta comunidad en las lavas del Cabo de Gata (Almería, España).

Esta localidad es, hasta ahora, el límite septentrional y oriental de la comunidad en el Norte de África y amplía considerablemente su área de distribución conocida.

\section{* Ramalinetum bourgeanae Llimona \& Egea, 1984}

Comunidad indicadora de lugares áridos pero con elevada humedad atmosférica. Ocupa las aristas de rocas soleadas y eutrofizadas por las deyecciones de aves. 
Se ha localizado en el Cabo Tres Forcas, constituida fundamentalmente por tres especies (Ramalina bourgeana (4.4), Xanthoria aureola (2.2) y Buellia cerussata (3.4) que ocupan prácticamente el $100 \%$ de la superficie colonizada.

\section{* Parmelietum tinctinae Egea \& Llimona, 1987}

Se instala sobre superficies soleadas poco o nada inclinadas, muy eutrofizadas y próximas al suelo.

En el Cabo Tres Forcas, la asociación se caracteriza por la presencia de Parmelia tinctina (3.3), P. pulla (3.3) y P. verruculifera (1.1), junto a otras especies nitrófilas como Xanthoria aureola (2.2), Acarospora umbilicata (2.2), Caloplaca festiva (1.1), C. irrubescens (1.1), C. subpallida (1.1), etc.

En el Cabo Falcón la asociación se encuentra de forma fragmentaria. A destacar la ausencia de Parmelia tinctina y P. verruculifera y la entrada de otras especies frecuentes en este tipo de comunidades, como Caloplaca heppiana (1.1), Toninia aromatica (+), Lecanora muralis (+), Verrucaria lecideoides (1.1), etc.

\section{* Pertusarietum gallicae Llimona \& Egea 1987}

Coloniza las paredes verticales o subverticales orientadas al $\mathrm{N}$ o al $\mathrm{W}$, así como las superficies poco o nada inclinadas, en lugares protegidos de las radiaciones solares directas e iluminadas. Si la humedad atmosférica es elevada puede instalarse en lugares más o menos soleados. En estas últimas condiciones se encuentra siempre empobrecida.

En el cabo Tres Forcas y Feknana la asociación se encuentra en su óptimo desarrollo, con una composición florística semejante a la observada en el Cabo de Gata (Almería) y Cabo de Palos (Murcia). Junto a Pertusaria gallica (2.3) se encuentran Ramalia clementeana (+), Rinodina subglaucescens (2.2), Buellia cerussata (2.2), B. subdisciformis (1.2), Lecanora montagnei (1.1), L. schistina (1.2) y Diploicia subcanescens (+). En ocasiones, penetra en la comunidad Pertusaria monogona (1.4) que caracteriza a la subas. pertusarietosum monogonae Llimona \& Egea 1984, indicadora de condiciones locales de elevada humedad nocturna.

En Los Andaluces, la comunidad inventariada corresponde a esta última subasociación, y se encuentra ya algo empobrecida. Junto a Pertusaria gallica (1.2) y Pertusaria monogona (2.2) Buellia subdisciformis (+), Lecanora schistina (3.4), Caloplaca festiva $(+) \mathrm{y}$, en ocasiones, entra en ella Dirina schistosa (1.1) como transgresiva.

\section{$\star$ Dirinetum schistosae Egea \& Llimona, 1984}

Comunidad ombrófoba de paredes verticales o superverticales y paredes y techos de pequeñas cuevas excavadas en la roca.

En el Cabo Tres Forcas y Feknana la asociación presenta la misma composición florística que en el SE de España (EGEA \& LLIMONA, 1984). Se caracteriza por la presencia de Dirina repanda var. schistosa (3.3), D. repanda var. schistosa f. ${ }^{a}$. sorediata (1.1), Lecanactis monstrosa (1.1), Opegrapha lutulenta (1.1), Roccella phycopsis (+) y Diploicia subcanescens (2.2). Junto a estas son frecuentes Lecanora schistina (2.2), Buellia subdisciformis (1.1) y Rinodina subglaucescens (1.1).

En Los Andaluces y Cabo Falcón, la comunidad se ve enriquecida con la entrada de Thelopsis isiaca (1.1) y, en las lavas con una cierta costra de carbonatos en superficie, Opegrapha durieuri (2.2). En Los Andaluces también hemos detectado una comunidad próxima a la asociación Roccelletum canariensis (Follman, 1976), sobre superficies verticales sombreadas, constituida por: Roccella canariensis (2.2), R. vicentina $(2.2), R$. phycopsis $(+)$, Ramalina bourgeana (+), Dirina repanda var. schistosa (3.3) y Thelopsis isiaca (+). 


\section{CATÁLOGO FLORÍSTICO}

Los resultados florísticos obtenidos en este estudio pueden verse en la tabla I. Los números que figuran en las columnas de la derecha corresponden a las localidades donde se han recolectado los ejemplares.

Desde el punto de vista ecológico y corológico son de destacar las siguientes especies.

\section{TABLA I}

Acarospora heufleriana Körber

Acarospora microcarpa (Nyl.) Wedd.

Aspicilia calcarea (L.) Mudd

Aspicilia hoffmannii (Ach.) Flagey

Aspicilia intermutans (Nyl.) Arnold

Aspicilia radiosa (Hoffm.) Poelt \& Leuckert

Buellia caloplacivora Llimona \& Egea

Buellia cerussata Llimona \& R.G. Werner

Buellia glaucoatra (Nyl) Clauzade

Buellia punctata (Hoffm.) Massal.

Buellia subdisciformis (Leighton) Vainio

Caloplaca festiva (Ach.) Zwackh

Caloplaca festiva (Ach.) Zwackh

var. depauperata $\mathrm{H}$. Magn.

Caloplaca gloriae Llimona \& R.G. Werner

Caloplaca heppiana (Müll. Arg.) Zahlbr.

Caloplaca irrubescens (Nyl) Zahlbr.

Caloplaca subpallida $\mathrm{H}$. Magn.

Candelariella aurella (Hoffm.) Zahlbr.

Candelariella vitellina (Ehrh.) Müll. Arg.

Catillaria chalibeya (Borrer) Massal.

Collema tenax (Swartz) Ach. em. Degel.

Dermatocarpon miniatum (L.) Mann

Dimelaena radiata (Tuck.) Hale \& Culb.

Diploicia subcanescens (R.G. Werner) Hafellner

Diploschistes actinostomus (Pers.) Zahlbr.

Dirina repanda (Ach.) Fr. var. schistosa Bagl.

Dirina repanda $\mathrm{f}^{\mathrm{a}}$ sorediata Roux \& Llimona

Lecanactis grumulosa (Dufour) Fr.

Lecanactis monstrosa Bagl.

Lecanactis patellaroides (Nyl.) Vainio

Lecania spadicea Flotow var. gennarii (Bagl.) Steiner

Lecania turicensis (Hepp.) Müll. Arg.

Lecanora albescens (Hoffm.) Floerke

Lecanora dispersa (Pers.) Röhl

Lecanora gangaleoides Nyl.

Lecanora montagnei (Fr.) Schaerer

Lecanora schistina (Nyl.) Arnold

Lecidella subincongrua $(\mathrm{Nyl})$ Hertel \& Leuckert

Lichinella stipatula $(\mathrm{Nyl})$

Opegrapha durieui Mont.

Opegrapha lutulenta $\mathrm{Nyl}$.

Opegrapha mougeotii Massal.

Opegrapha subelevata Nyl. 
Opegrapha trifurcata Hepp

Opegrapha werneri Faurel, Ozenda \& Schotter

Parmelia pulla Ach.

Parmelia tinctina Maheu \& Gillet

Parmelia verruculifera $\mathrm{Nyl}$.

Peltula euploca (Ach.) Poelt ex Pisút

Pertusaria gallica B. de Lesd.

Pertusaria monogona Nyl.

Polysporina simplex (Davies) Vezda

Porina linearis (Leighton) Zahlbr.

Ramalina bourgeana (Mont.) Nyl.

Ramalina clementeana Llimona \& R.G. Werner

Rhizocarpon viridiatrum (Wulf.) Körber

Rinodina subglaucescens (Nyl.) Sheard

Roccella canariensis Davies em Vainio

Roccella phycopsis (Ach.) Ach.

Roccella vicentina (Vainio) Vainio

Solenopsora holophaea (Mont.) G. Samp.

Thelopsis isiaca Stizenb.

Toninia aromatica $(\mathrm{Sm}$.) Massal.

Verrucaria lecideoides (Masal.) Trev.

Verrucaria nigrescens Pers.

Xanthoria aureola (Ach.) Floerke

Xanthoria resendei (Link.) Th. Fr.

\section{Acarospora heufleuriana Körber}

Termófilo, heliófilo, fotófilo y nitrófilo. Óptimo en las zonas térmicas de la Región Mediterránea occidental y Macaronésica. En Marruecos señalado con anterioridad de Aït Benhaddou, Anti Atlas, a 1500 m (WERNER, 1948).

\section{Buellia caloplacivora Llimona \& Egea}

Descrito de las lavas del SE de España, sobre el talo de Caloplaca littorea (LLIMONA \& EGEA, 1984). Se ha encontrado posteriormente en Tenerife (EGEA \& col., 1987) sobre Caloplaca marina y ahora en el Cabo Falcón, donde invade el talo de diversas especies de Caloplaca.

\section{Buellia cerussata Llimona \& R.G. Werner}

Termófilo, de heliófilo a heliófobo, fotófilo, ombrófilo. En África señalado con anterioridad de Tánger, Marruecos (EGEA \& RowE, 1987).

\section{Caloplaca gloriae Llimona \& R.G. Werner}

Muy xerófilo, termófilo, heliófilo, nitrófilo y más o menos acidófilo. Sólo se ha encontrado sobre lavas próximas al litoral, en el SE de España, Canarias y SW de Marruecos (LLIMONA \& WERNER, 1975). Nueva cita para Argelia.

Dimelaena radiata (Tuck.) Hale \& Culb.

Muy xerófilo, termófilo, heliófilo. Sobre rocas ácidas próximas al litoral en lugares áridos y subáridos de clima mediterráneo (SE de España, SW de Marruecos, Canarias y California). Nueva cita para Argelia. 


\section{Diploicia subcanescens (R.G. Werner) Hafellner \& Poelt}

Xerófilo, termófilo, indiferente a la exposición y luminosidad, aunque se comporta mayormente como heliófobo, más o menos fotófilo y ombrófilo. Óptimo en el litoral de la Región Mediterránea occidental y Macaronésica. Nueva cita para Argelia.

Lecania spadicea (Flotow) Zahlbr. var. gennarii (Bagl.) Steiner

Termófilo, heliófilo, fotófilo, nitrófilo. Se instala sobre rocas calizas del litoral de la Región Mediterránea. Nueva cita para Argelia.

\section{Lecanora montagnei (Fr.) Schaerer}

Termófilo, heliófilo, fotófilo, ombrófilo. Óptimo en zonas térmicas y subáridas de la Región Mediterránea occidental. Penetra en algunos puntos de ombroclima subhúmedo (Cádiz, Tánger) y llega a la Región Macaronésica (EGEA \& col. 1987).

\section{Opegrapha werneri Faurel, Ozenda \& Schotter}

Taxon fácilmente reconocible por su talo rodeado de un hipotalo negro muy marcado. Ascocarpos lirelinos hundidos o semihundidos en el talo. Esporas de $28-36 \times(3) 4-5 \mu \mathrm{m}$, (35-42 $\times$ 4-6 $\mu \mathrm{m}$, según Faurel \& col. 1953), con 7-11 septos. Conidios curvados de $15-18 \times 1-1,5 \mu \mathrm{m}$ y talo $\mathrm{Cl}+$ rojo carmín. Este último carácter no es mencionado por FAUREL \& col. (1953), en la descripción original.

Citado hasta la fecha solamente en Alger, cerca de Icosium, en el Bosque de Baihem, sobre la pared norte del «Grand Rocher».

Pertusaria gallica B. de Lesd.

Termófilo, heliófilo, fotófilo, ombrófilo. En África señalado con anterioridad de Tánger, Marruecos (EGEA \& RowE, 1987)

\section{Ramalina clementeana Llimona \& R.G. Werner}

Termófilo, heliófilo, fotófilo, ombrófilo. Citado hasta la fecha solamente del SE de España (LLIMONA \& WERNER, 1975; EGEA \& LLIMONA, 1983).

Roccella canariensis Davies em. Vainio

Aerohigrófilo, termófilo, heliófilo, fotófilo y más o menos ombrófolo. Óptimo en la Región Macaronésica, penetra en algunos puntos de la Región Mediterránea (Marruecos, Portugal). Nueva cita para Argelia.

\section{Bibliografía}

BENABID, A. (1984). Étude phytoecologique des peuplements forestiers et preforestiers du Rif centrooccidental (Maroc). Travaux de l'Institut Scientifique (Serie Botanica) 34: 1-64. Rabat.

EgEA, J. M. \& X. Llimona (1982). Los líquenes silicícolas de la Sierra del Cabo de Palos; estudio florístico, fitosociológico y ecológico. Acta Bot. Malacitana 7: 11-38.

EgEA, J. M. \& X. LLimona (1983). Mapas de distribución en el SE de España de los principales líquenes silicícolas. I. Anal. Univer. Murcia, Ciencias, 41 (1-4): 209-219.

EGEA, J. M. \& X. Llimona (1984). Las comunidades liquénicas ombrófobas de la costa del SE de España, comprendidas entre el Penyal D'Ifac (Alicante) y Almería. Collect. Bot. (Barcelona) 15: 205-219. 
EgEA, J. M. \& X. Llimona (1987). Comunidades liquénicas de las rocas silíceas del SE de España. Acta Botanica Barcinonensia 36: 1-123.

EGEA, J.M. \& J. G. RowE (1987). Prospecciones liquenológicas en África del Norte. I. Líquenes silicícolas de Marruecos. Collect. Bot. (Barcelona) 17(1): 27-45.

EgEA, J. M., C. HERnÁNDEZ-PADRÓn \& X. Llimona (1987). Aportación al conocimiento de las comunidades de líquenes saxícolas de los pisos inferiores de Tenerife (Canarias). Butll. Inst. Catalana Hist. Nat. 54 (Sec. bot., 6): 37-53.

FAurel, L., P. Ozenda \& G. SchotTer (1953). Notes lichenologiques Nord-Africaines. Bull. Soc. Hist. Nat. Afrique du Nord. 44: 367-384.

Follman, G. (1976). Lichen flora and lichen vegetation of the Canary Island, 267-286. In KunKel, G. (Ed.) Biogeography and Ecology in the Canary Island. Dr. W. Junk B.V., The Hague.

Llimona, X. (1975). Xanthoria resendei Poelt et Tav. en el SE de España; Fitosociología y Corología. Anales Inst. Bot. Cavanilles 32(2): 909-922.

Llimona, X. \& J. M. EgEA (1984). La vegetación liquénica saxícola de los volcanes del Mar Menor. (Murcia, SE de España). Butll. Inst. Catalana Hist. Nat. 51 (Sec. Bot. 5): 77-99.

LLimona, X. \& Werner R. G (1975). Quelques lichens nouveaux ou intéressants de la Sierra de Gata (Almería SE de España). Acta Phytotax. Barcinonensia, 16: 1-24.

WERNER, R. G. (1948). Les origines de la flore cryptogamique du Maroc d'après nos connaissances actuelles. Vol. Jubil. Soc. Sci. Nat. Maroc. 1920-1945: 147-202. 